\title{
Assistive technology designed to support self-management of people with dementia: user involvement, dissemination, and adoption. A scoping review
}

\author{
Laila Øksnebjerg, ${ }^{1}$ (1) Janet Janbek, ${ }^{1}$ Bob Woods, ${ }^{2}$ and Gunhild Waldemar ${ }^{1}$ \\ ${ }^{1}$ Danish Dementia Research Centre, Department of Neurology, Rigshospitalet, University of Copenhagen, Copenhagen, Denmark \\ ${ }^{2}$ Dementia Services Development Centre Wales, Bangor University, Bangor, UK
}

Background: Assistive technology is advocated as a key solution to the need for support among people living with dementia. There is growing awareness of the benefits of user involvement in the design and test of these technologies and the need to identifying applicable and effective methods for implementation. The aim of this review was to explore and synthesize research addressing assistive technology designed to be used by people with dementia for self-management. Further research aims were to explore if and how user involvement, dissemination, and adoption of assistive technology were addressed.

Method: Electronic databases were searched using specified search terms. Key publications and grey literature sources were hand-searched. Materials published until year end 2018 were included. The results were summarized according to the research aims.

Results: Eleven papers derived from eight studies were included. The studies presented data from prototype design and testing, and the review showed great variation in study scope, design, and methodology. User involvement varied from extensive involvement to no user involvement. Methods for adoption also varied widely and only targeted prototype testing. None of the studies addressed dissemination.

Conclusion: The results of this review underline the need for well-designed high-quality research into all the aspects that are essential to deliver applicable, effective, and sustainable assistive technology to support self-management of people with dementia. There is a need for evidence-based methods to promote and qualify user involvement, dissemination, and adoption. The results also point to the need for standardized outcome measures and standards for conducting and reporting research to improve its quality and impact.

Key words: Dementia, activities of daily living (ADLs), cognitive disorders, internet-based interventions, psychosocial interventions

\section{Introduction}

The number of people living with dementia worldwide is fast growing (World Health Organisation, 2017), and there is a strong demand to take action and move forward pervasive and innovative solutions to meet their needs for support and care. In recent years, the rapid advances in information technology and digitalization have attracted political attention as a key solution to these challenges (Alzheimer's Disease International, 2016; World Health Organisation,

Correspondence should be addressed to: Laila Øksnebjerg, Danish Dementia Research Centre, Department of Neurology, University of Copenhagen, Rigshospitalet, Copenhagen, Denmark. Phone: 00452225 3335. Email: laila .oeksnebjerg.02@regionh.dk. Received15 Apr 2019; revision requested 14 Jun 2019; revised version received 04 Jul 2019; accepted 09 Oct 2019. First published online 25 November 2019.
2017), and we see a rapid growth in assistive technology (AT) being promoted as suitable and effective solutions for people with dementia (Asghar et al., 2017; Gibson et al., 2016). AT is an umbrella term encompassing "Any item, piece of equipment, software program, or product system that is used to increase, maintain or improve the functional capabilities of persons with disabilities" (Assistive Technology Industry Association, 2019). This review specifically addresses electronically powered AT.

One perspective given special attention is the potential of AT to promote the capacity of people with dementia and support them in coping with cognitive deficits and managing everyday life as independently as possible, e.g. electronic calendars, prompting and reminder devices, and navigation aids (Gibson et al., 2015; Gillespie et al., 2012; 
Kenigsberg et al., 2017; Meiland et al., 2017). These solutions can be conceptualized within the framework of self-management as defined by Barlow et al. (2002) as “... individual's ability to manage the symptoms, treatment, physical and psychosocial consequences and life style changes inherent in living with a chronic condition".

However, despite the optimism that AT is a core solution to the current and future challenges in dementia support and care, there is also increasing awareness of the challenges that impede significant progress within this field. For instance, many technologies are still underdeveloped (Knapp et al., 2015; Meiland et al., 2017), and the many psychosocial and societal factors that influence the adoption of AT are not addressed (Knapp et al., 2015; Meiland et al., 2017; Smith et al., 2018). Lack of adoption or early abandonment of technology addressing health-related issues is a well-known problem both in nonclinical and clinical populations (Federici et al., 2016; Krebs and Duncan, 2015; van Gemert-Pijnenet al., 2014). It, therefore, follows that a special effort is required when designing and implementing AT for people with dementia who have special needs due to cognitive decline, and who might also be experiencing age-related constraints such as hearing, visual, and other physical disabilities. Generational factors can also influence response to AT solutions. To make progress within the field of AT supporting self-management of people with dementia, we need to identify which factors facilitate or impede the adoption and continued use of AT.

Recent reviews and position papers, addressing AT for people with dementia from a broad perspective, generally come to concurrent conclusions regarding a range of topics to be addressed in order to make advances in research and bring to market evidence-based solutions that are usable and effective in everyday lives of people with dementia (Bächle et al., 2018; Holthe et al., 2018; Ienca et al., 2017; Kenigsberg et al., 2017; King and Dwan, 2017; Meiland et al., 2017; Robillard et al., 2018). Among the most recurrent is the recommendation to involve people with dementia in the design and testing of AT, to make sure that their needs, preferences, and capacities are met and also to pursue this end user perspective when developing applicable and effective methods for dissemination and adoption of AT.

These recent reviews and position papers also reveal that research addressing AT for people with dementia is still limited and rather heterogeneous and is generally not characterized by randomized controlled trials (van der Roest et al., 2017). Based on these preconditions, a scoping review methodology was selected for this review.
This scoping review was not conducted to compile an exhaustive catalogue of the broad variety of AT promoted for people with dementia. Rather, our key focus was to explore the characteristics of studies where AT was designed to be used by people with dementia for self-management in an autonomous and flexible manner, to support their independence in management of everyday life. Furthermore, we wanted to analyze if and how studies had involved users in the design and/or test of this $\mathrm{AT}$, and the results of this involvement. We also wanted to investigate studies that had addressed methods for implementation of AT and/or adoption by the end users.

Accordingly, the research aims of the scoping review were to:

1. Synthesize data from studies where AT had been designed to support self-management of people with dementia.

2. Explore if people with dementia had been included in the design and/or test of this AT, and if so describe methods and results of this involvement.

3. Explore if issues of dissemination and adoption of AT had been addressed, and if so describe the methods used.

\section{Methods}

The scoping review was conducted and reported according to the PRISMA Extension for Scoping Reviews (Tricco et al., 2018) and guided by the recommendations on conducting systematic scoping reviews from Peters et al. (2015).

\section{Data sources and search strategy}

First, data were derived from systematic searches in the electronic databases PubMed, PsycINFO, Web of Science, Scopus, Embase, and CINAHL. A broad search strategy was designed to ensure that the inclusion of studies was as comprehensive as possible. Search terms were derived from titles, abstracts, and keywords identified in key publications and from search terms used in previous reviews related to the scope of this review.

The following search terms were used: Dementia (Dementia or Alzheimer Disease) and AT, supportive technology, self-help devices, information and communication technology, information technology, cognitive prosthetics, electronic memory aids, self-help devices, self-help technology, or reminder systems. $\mathrm{MeSH}$ terms and truncation of search terms were used where appropriate. Details on search terms and databases are specified in Table 1.

To locate additional materials, reference lists of key publications, e.g. reviews and position papers, 
Table 1 Search terms and results, literature for review December 18, 2018

\begin{tabular}{|c|c|c|}
\hline \multicolumn{3}{|c|}{ SEARCH TERMS AND RESULTS } \\
\hline SUBJECT & $\begin{array}{l}\text { UNIFIED SEARCH TERM } \\
\text { (INCLUDING BOOLEAN OPERATORS) }\end{array}$ & RESULTS \\
\hline Participants & $\begin{array}{l}\text { Dementia }[\mathrm{MeSH}] \text { OR Alzheimer } \\
\text { Disease }[\mathrm{MeSH}] \text { OR Alzheimer }\end{array}$ & $\begin{array}{l}\text { Pubmed: } 207.270 \\
\text { PsycINFO: } 64.200 \\
\text { Web of Sciences: } 230.488 \\
\text { SCOPUS: } 730.437 \\
\text { EMBASE: } 323.591 \\
\text { CINAHL: } 35.261\end{array}$ \\
\hline 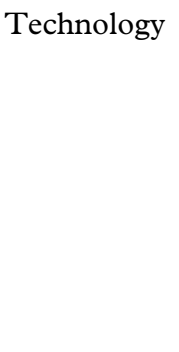 & $\begin{array}{l}\text { Technology (assist }{ }^{*} \text { OR support }{ }^{*} \text { ) } \\
\text { OR Self-Help Devices [MeSH] } \\
\text { OR Information and communication } \\
\text { technology OR ICT OR Information } \\
\text { Technology [MeSH] OR Cognitive } \\
\text { prosthetics OR Electronic memory aid* } \\
\text { OR Self-help devices [MeSH] OR } \\
\text { Self-help technology OR Reminder } \\
\text { Systems [MeSH] }\end{array}$ & $\begin{array}{l}\text { Pubmed: } 26.674 \\
\text { PsycINFO: } 8.267 \\
\text { Web of Sciences: } 1.250 .039 \\
\text { SCOPUS: } 11.507 \\
\text { EMBASE: } 11.500 \\
\text { CINAHL: } 1.758\end{array}$ \\
\hline Unifying the & h: participants AND Technology & $\begin{array}{l}\text { Pubmed: } 371 \\
\text { PsycINFO: } 83 \\
\text { Web of Sciences: } 272 \\
\text { SCOPUS: } 9.105\end{array}$ \\
\hline
\end{tabular}

were hand-searched. In addition, to locate additional grey literature, Opengrey was searched by the keywords dementia and technology.

To avoid missing essential data at the stage of full-text screening, an additional search was conducted if a reference was about to be excluded because of insufficient information to explore the review aims. In these cases, author names and study names/acronyms were searched in Google and Google Scholar. If further publications were located, these were included in the full-text screening process.

The data search was conducted in December 2018, and all records published up until then were included, with no start date applied.

\section{Inclusion and exclusion criteria}

\section{Materials}

This scoping review was conducted to explore the present state of research; hence, a wide range of data were accepted for inclusion covering all kinds of material published in peer-reviewed journals, book chapters, meeting abstracts, and trial registrations. Literature not presenting the original data from a research project, e.g. reviews and position papers, was excluded. Only material published in English was included.

\section{Population}

Studies related to people with dementia were included, and there were no limits with regard to etiology or severity of disease. Studies involving people with mild cognitive impairment were only included if people with dementia were also included. Studies only addressing caregivers (family and/or professional) or other groups of end users were excluded.

\section{Interventions}

To investigate the first aim, studies eligible for this review included results from the design process and/ or test of hardware or software designed for people with dementia and the main user of the technology was the person with dementia, with support from caregivers if needed. The scope of the technology was to support self-management of people with dementia, e.g. through support of cognitive functions (memory, spatial orientation, communication, etc.), or through holistic solutions addressing support of self-management in various ways. Consequently, the following types of AT were excluded: AT supporting specific psychosocial intervention, e.g. reminiscence or cognitive stimulation therapy, AT used to support education or provide information, and AT used for cognitive training and technology used for physical exercise. In addition, studies were also excluded if they only addressed one specific and predefined activity, e.g. medication management or handwashing. Technology that was only designed to support security by surveillance, e.g. GPS trackers or sensor technology, was also excluded. However, in many instances, AT includes 


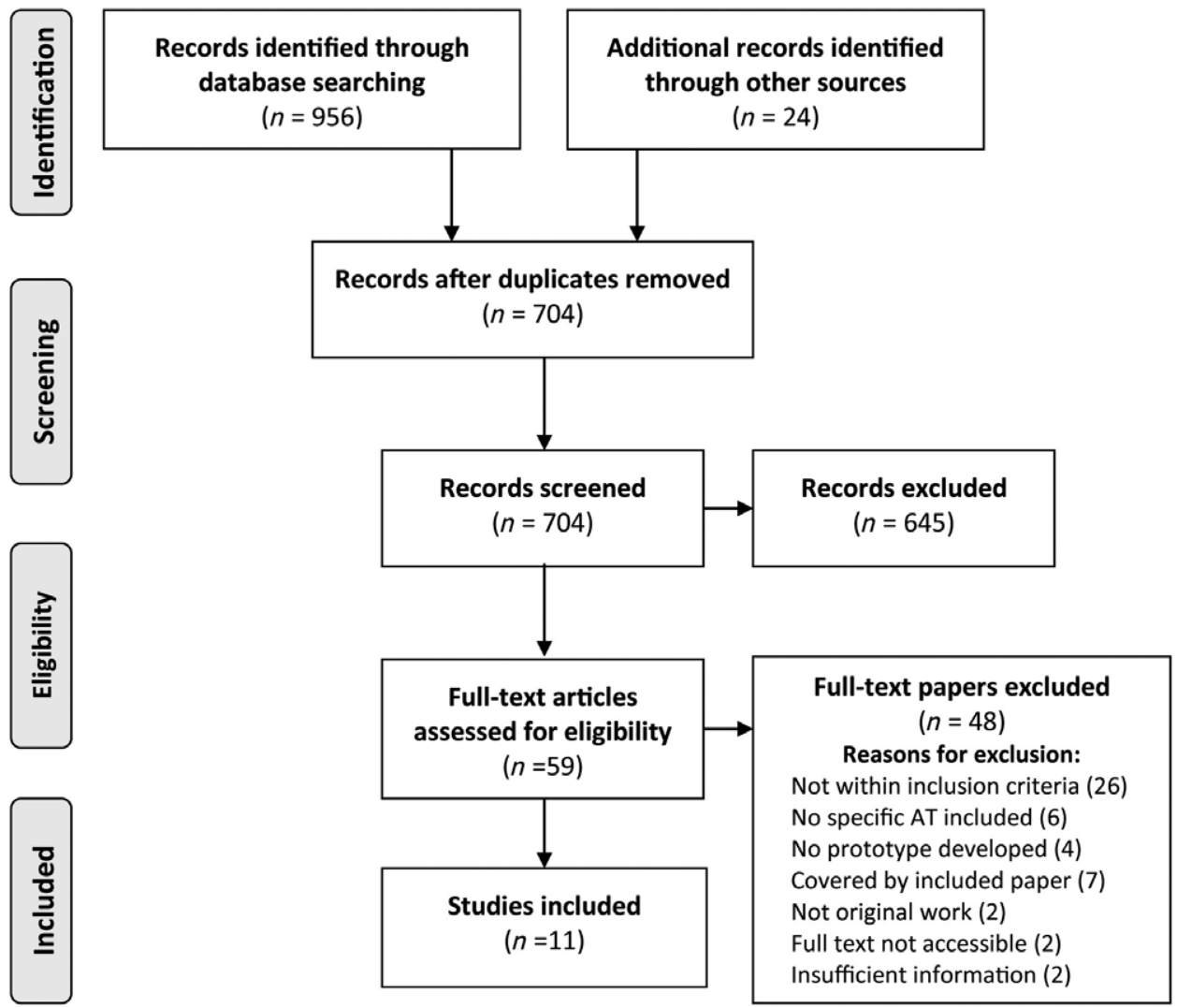

Figure 1. Prisma flow diagram illustrating the study selection process.

several features/functionalities and if the main scope for the AT was within the inclusion criteria of this review, and additional features were not, the study was included, e.g. in cases where GPS was an additional feature.

To further address the second aim, concerning the involvement of people with dementia in the design and/or test of AT, studies that fulfilled criteria for the first research aim were further reviewed. They were included in this part of the review if they addressed any kind of involvement of people with dementia in the scoping, design, or test of the technology. To include the broadest possible range of data, studies were also included if they had byproxy involvement of end users, for instance involvement of caregivers.

Finally, exploring the third aim of the review, studies that fulfilled criteria of the first research aim were further reviewed and included in this part of the review if they involved activities addressing adoption and/or dissemination of AT to people with dementia.

\section{Study selection}

The search and selection procedures, and reasons for exclusion, were recorded and are summarized in
Figure 1. Two of the authors (first and second author) assessed the eligibility of all studies independently and in a standardized manner. Covidence (Covidence.org) was used for review management and the blinded review procedure. First, the 704 retrieved records from the search were screened by title and abstract. Second, 59 full texts were assessed for eligibility according to the criteria described above. Disagreements were discussed and resolved among the reviewers.

According to the nature of this scoping review, studies were not selected based on methodological quality, and no quality assessment was performed.

\section{Data extraction}

As listed in Table 2, the following information was extracted from the included studies: Publication details (authors, year of publication, title and origin). To address the first research aim, the aim and features of the AT were recorded. To address the second research aim, characteristics of participants, methods for user involvement, and evaluation methods and results were recorded. Finally, to address the third research aim, methods for adoption and/or dissemination were recorded. 
Table 2 Characteristics and results from the studies included in the review

\begin{tabular}{|c|c|c|c|c|c|c|c|c|}
\hline \multirow[b]{3}{*}{ STUDY } & \multirow[b]{3}{*}{$\begin{array}{l}\text { PUBLICATION } \\
\text { AND ORIGIN }\end{array}$} & \multicolumn{2}{|c|}{ REVIEW AIM 1} & \multicolumn{3}{|c|}{ REVIEW AIM 2} & \multicolumn{2}{|c|}{ REVIEW AIM 3} \\
\hline & & \multicolumn{2}{|c|}{$\begin{array}{c}\text { ASSISTIVE TECHNOLOGY USED BY } \\
\text { PEOPLE WITH DEMENTIA FOR } \\
\text { SELF-MANAGEMENT }\end{array}$} & \multicolumn{3}{|c|}{$\begin{array}{l}\text { INVOLVEMENT OF END USERS IN } \\
\text { DESIGN AND/OR TEST PHASES }\end{array}$} & \multicolumn{2}{|c|}{$\begin{array}{l}\text { METHODS ADDRESSING } \\
\text { ADOPTION AND } \\
\text { DISSEMINATION }\end{array}$} \\
\hline & & $\begin{array}{l}\text { AIM OF } \\
\text { TECHNOLOGY } \\
\text { AND TARGET } \\
\text { USERS }\end{array}$ & FEATURES & PARTICIPANTS & $\begin{array}{l}\text { METHODS } \\
\text { FOR USER } \\
\text { INVOLVEMENT }\end{array}$ & $\begin{array}{l}\text { EVALUATION } \\
\text { METHODS AND } \\
\text { RESULTS }\end{array}$ & ADOPTION & DISSEMINATION \\
\hline COGKNOW & $\begin{array}{l}\text { Meiland et al. } \\
\text { (2007) } \\
\text { Origin: The } \\
\text { Netherlands, } \\
\text { Ireland and } \\
\text { Sweden } \\
\text { Meiland et al. } \\
\text { (2012) } \\
\text { Origin: The } \\
\text { Netherlands, } \\
\text { Ireland and } \\
\text { Sweden }\end{array}$ & $\begin{array}{l}\text { Aim: Supporting } \\
\text { daily function } \\
\text { through support } \\
\text { of memory, } \\
\text { social contacts, } \\
\text { daily activities, } \\
\text { and safety. } \\
\text { Target users: } \\
\text { People with } \\
\text { mild dementia }\end{array}$ & $\begin{array}{l}\text { The holistic } \\
\text { solution } \\
\text { included } \\
\text { two user } \\
\text { devices: } \\
\text { - Stationary } \\
\text { touch screen } \\
\text { - Mobile device } \\
\text { And: } \\
\text { - Home-based } \\
\text { sensors } \\
\text { - Actuators }\end{array}$ & $\begin{array}{l}\text { Design phase: } \\
17 \text { dyads: } \\
\text { people with } \\
\text { AD and FC. } \\
\text { Details verifying } \\
\text { diagnosis: Yes. } \\
\text { Test phase: } 42 \\
\text { dyads: People } \\
\text { with AD and FC. } \\
\text { Details verifying } \\
\text { diagnosis: Yes. }\end{array}$ & $\begin{array}{l}\text { Test phase: } \\
\text { Home testing. } \\
\text { Duration: Three } \\
\text { successive test } \\
\text { cycles: } 0.5 \text { days, } \\
1 \text { week and } 3- \\
8 \text { weeks. }\end{array}$ & $\begin{array}{l}\text { Design phase: } \\
\text { A prototype was } \\
\text { designed } \\
\text { based on the } \\
\text { results } \\
\text { from workshops } \\
\text { and interviews. } \\
\text { Test phase: } \\
\text { - Interviews } \\
\text { - Questionnaires } \\
\text { - Observations } \\
\text { - Diaries } \\
\text { - Data log } \\
\text { - Standardized } \\
\text { outcome measures, } \\
\text { e.g. autonomy, } \\
\text { coping, and quality } \\
\text { of life. } \\
\text { Results: No } \\
\text { statistically significant } \\
\text { differences on } \\
\text { standardized } \\
\text { outcome measures. } \\
\text { Authors conclude: } \\
\text { The solution was } \\
\text { overall rated as } \\
\text { user-friendly and } \\
\text { useful. Effectiveness } \\
\text { of the system in daily } \\
\text { life could not be } \\
\text { evaluated due to } \\
\text { insufficient duration } \\
\text { of the test period. }\end{array}$ & $\begin{array}{l}\text { User manual } \\
\text { and } \\
\text { helpdesk. }\end{array}$ & N/A \\
\hline
\end{tabular}


Table 2 Continued

\begin{tabular}{|c|c|c|c|c|c|c|c|c|}
\hline \multirow[b]{3}{*}{ STUDY } & & \multicolumn{2}{|c|}{ REVIEW AIM 1} & \multicolumn{3}{|c|}{ REVIEW AIM 2} & \multicolumn{2}{|c|}{ REVIEW AIM 3} \\
\hline & \multirow[b]{2}{*}{$\begin{array}{l}\text { PUBLICATION } \\
\text { AND ORIGIN }\end{array}$} & \multicolumn{2}{|c|}{$\begin{array}{c}\text { ASSISTIVE TECHNOLOGY USED BY } \\
\text { PEOPLE WITH DEMENTIA FOR } \\
\text { SELF-MANAGEMENT }\end{array}$} & \multicolumn{3}{|c|}{$\begin{array}{l}\text { INVOLVEMENT OF END USERS IN } \\
\text { DESIGN AND/OR TEST PHASES }\end{array}$} & \multicolumn{2}{|c|}{$\begin{array}{l}\text { METHODS ADDRESSING } \\
\text { ADOPTION AND } \\
\text { DISSEMINATION }\end{array}$} \\
\hline & & $\begin{array}{l}\text { TECHNOLOGY } \\
\text { AND TARGET } \\
\text { USERS }\end{array}$ & FEATURES & PARTICIPANTS & $\begin{array}{l}\text { METHODS } \\
\text { FOR USER } \\
\text { INVOLVEMENT }\end{array}$ & $\begin{array}{l}\text { EVALUATION } \\
\text { METHODS AND } \\
\text { RESULTS }\end{array}$ & ADOPTION & DISSEMINATION \\
\hline Rosetta & $\begin{array}{l}\text { Meiland et al. } \\
\text { (2014) } \\
\text { Origin: The } \\
\text { Netherlands } \\
\text { and Germany }\end{array}$ & $\begin{array}{l}\text { Aim: Supporting } \\
\text { daily function } \\
\text { through support } \\
\text { of memory, } \\
\text { social contacts, } \\
\text { daily activities } \\
\text { and safety. } \\
\text { Target users: } \\
\text { people with } \\
\text { dementia }\end{array}$ & $\begin{array}{l}\text { The holistic solution } \\
\text { included three } \\
\text { kinds devices/ } \\
\text { hardware: } \\
\text { - Day navigator: } \\
\text { video home } \\
\text { terminal and } \\
\text { mobile device } \\
\text { supporting } \\
\text { memory, social } \\
\text { contacts, daily } \\
\text { activities and } \\
\text { safety. } \\
\text { - Detection system: } \\
\text { monitor the status } \\
\text { of the user. }\end{array}$ & $\begin{array}{l}\text { Design phase: } \\
11 \text { PwD, } 3 \text { MCI, } \\
13 \text { FC, } 23 \\
\text { professionals. } \\
\text { Specific details } \\
\text { verifying } \\
\text { diagnosis: No, } \\
\text { but from } \\
\text { dementia care } \\
\text { organizations. }\end{array}$ & $\begin{array}{l}\text { Design phase: } \\
\text { Workshops, } \\
\text { individual } \\
\text { interviews, } \\
\text { expert } \\
\text { meetings, } \\
\text { consultations } \\
\text { and prototype } \\
\text { testing. }\end{array}$ & $\begin{array}{l}\text { Design phase: } \\
\text { A prototype was } \\
\text { designed based } \\
\text { on the results } \\
\text { from the iterative } \\
\text { design process. }\end{array}$ & N/A & N/A \\
\hline
\end{tabular}


Table 2 Continued

\begin{tabular}{|c|c|c|c|c|c|c|c|c|}
\hline \multirow[b]{3}{*}{ STUDY } & \multirow[b]{3}{*}{$\begin{array}{l}\text { PUBLICATION } \\
\text { AND ORIGIN }\end{array}$} & \multicolumn{2}{|c|}{ REVIEW AIM 1} & \multicolumn{3}{|c|}{ REVIEW AIM 2} & \multicolumn{2}{|c|}{ REVIEW AIM 3} \\
\hline & & \multicolumn{2}{|c|}{$\begin{array}{c}\text { ASSISTIVE TECHNOLOGY USED BY } \\
\text { PEOPLE WITH DEMENTIA FOR } \\
\text { SELF-MANAGEMENT }\end{array}$} & \multicolumn{3}{|c|}{$\begin{array}{l}\text { INVOLVEMENT OF END USERS IN } \\
\text { DESIGN AND/OR TEST PHASES }\end{array}$} & \multicolumn{2}{|c|}{$\begin{array}{c}\text { METHODS ADDRESSING } \\
\text { ADOPTION AND } \\
\text { DISSEMINATION }\end{array}$} \\
\hline & & $\begin{array}{l}\text { AIM OF } \\
\text { TECHNOLOGY } \\
\text { AND TARGET } \\
\text { USERS }\end{array}$ & FEATURES & PARTICIPANTS & $\begin{array}{l}\text { METHODS } \\
\text { FOR USER } \\
\text { INVOLVEMENT }\end{array}$ & $\begin{array}{l}\text { EVALUATION } \\
\text { METHODS AND } \\
\text { RESULTS }\end{array}$ & ADOPTION & DISSEMINATION \\
\hline $\begin{array}{l}\text { Rosetta } \\
\quad \text { (continued) }\end{array}$ & $\begin{array}{l}\text { Hattink et al. } \\
\text { (2016) } \\
\text { Origin: The } \\
\text { Netherlands, } \\
\text { Germany and } \\
\text { Belgium }\end{array}$ & & $\begin{array}{l}\text { - Surveillance } \\
\text { system: } \\
\text { detecting } \\
\text { emergency } \\
\text { situations. } \\
\text { The system } \\
\text { was an adapted } \\
\text { combination } \\
\text { of three previously } \\
\text { developed assistive } \\
\text { technology sys- } \\
\text { tems including the } \\
\text { COGKNOW. }\end{array}$ & $\begin{array}{l}\text { Test phase: } \\
42 \text { PwD or MCI } \\
32 \text { FC, } 6 \\
\text { professionals. } \\
\text { Specific details } \\
\text { verifying } \\
\text { diagnosis: yes }\end{array}$ & $\begin{array}{l}\text { Test phase: } \\
\text { Home test: } \\
\text { controlled } \\
\text { trial: partly } \\
\text { randomized } \\
\text { controlled trial } \\
\text { and partly } \\
\text { matched groups } \\
\text { design. } \\
\text { Duration: } \\
\text { 1/2-8 months. } \\
\text { Focus group } \\
\text { interview with a } \\
\text { subgroup of } \\
\text { family } \\
\text { caregivers. } \\
\text { Online } \\
\text { questionnaire } \\
\text { for professional } \\
\text { caregivers. }\end{array}$ & $\begin{array}{l}\text { Test phase: } \\
\text { - Interviews } \\
\text { - Questionnaires } \\
\text { - Standardized } \\
\text { outcome measures, } \\
\text { e.g. perceived } \\
\text { autonomy, quality } \\
\text { of life, care needs } \\
\text { and caregivers } \\
\text { feeling of competence. } \\
\text { Results: } \\
\text { No statistically } \\
\text { significant differences on } \\
\text { standardized } \\
\text { outcome measures. } \\
\text { Drop-out rate: } 18 \\
\text { Authors conclude: } \\
\text { Participants generally } \\
\text { found the system } \\
\text { useful for future care, but } \\
\text { user-friendliness was } \\
\text { generally } \\
\text { perceived low. }\end{array}$ & $\begin{array}{l}\text { Short } \\
\text { individual } \\
\text { introduction. }\end{array}$ & N/A \\
\hline
\end{tabular}


Table 2 Continued

\begin{tabular}{|c|c|c|c|c|c|c|c|c|}
\hline \multirow[b]{3}{*}{ STUDY } & & \multicolumn{2}{|c|}{ REVIEW AIM 1} & \multicolumn{3}{|c|}{ REVIEW AIM 2} & \multicolumn{2}{|c|}{ REVIEW AIM 3} \\
\hline & \multirow[b]{2}{*}{$\begin{array}{l}\text { PUBLICATION } \\
\text { AND ORIGIN }\end{array}$} & $\begin{array}{r}\text { ASSISTIVE TECH } \\
\text { PEOPLE WITH } \\
\text { SELF-MA }\end{array}$ & $\begin{array}{l}\text { NOLOGY USED BY } \\
\text { DEMENTIA FOR } \\
\text { NAGEMENT }\end{array}$ & $\begin{array}{r}\text { IN } \\
\text { D }\end{array}$ & $\begin{array}{l}\text { OLVEMENT OF E } \\
\text { SIGN AND/OR TI }\end{array}$ & $\begin{array}{l}\text { D USERS IN } \\
\text { ST PHASES }\end{array}$ & $\begin{array}{r}\text { METHODS } \\
\text { ADOPT } \\
\text { DISSEN }\end{array}$ & $\begin{array}{l}\text { ADDRESSING } \\
\text { ION AND } \\
\text { IINATION }\end{array}$ \\
\hline & & $\begin{array}{l}\text { TECHNOLOGY } \\
\text { AND TARGET } \\
\text { USERS }\end{array}$ & FEATURES & PARTICIPANTS & $\begin{array}{l}\text { METHODS } \\
\text { FOR USER } \\
\text { INVOLVEMENT }\end{array}$ & $\begin{array}{l}\text { EVALUATION } \\
\text { METHODS AND } \\
\text { RESULTS }\end{array}$ & ADOPTION & DISSEMINATION \\
\hline AP@LZ & $\begin{array}{l}\text { Imbeault et al. } \\
\quad(2014) \\
\text { Origin: Canada }\end{array}$ & $\begin{array}{l}\text { Aim: Compensation } \\
\text { for memory } \\
\text { problems in } \\
\text { day-to-day } \\
\text { activities. } \\
\text { Target users: } \\
\text { people with } \\
\text { Alzheimer's } \\
\text { disease. }\end{array}$ & $\begin{array}{l}\text { The holistic app } \\
\text { was designed for } \\
\text { smartphones and } \\
\text { included: } \\
\text { - Appointments } \\
\text { - Personal } \\
\text { information } \\
\text { - Medical } \\
\text { information } \\
\text { - Contacts } \\
\text { - Notepad }\end{array}$ & $\begin{array}{l}\text { Design phase: } \\
\text { No end users } \\
\text { were involved. } \\
\text { Test phase: } \\
\text { Two people AD. } \\
\text { Specific details } \\
\text { verifying } \\
\text { diagnosis: Yes. }\end{array}$ & $\begin{array}{l}\text { Design phase: } \\
\text { N/A } \\
\text { Test phase: } \\
\text { Laboratory } \\
\text { and home } \\
\text { testing. } \\
\text { Duration: } 11 \\
\text { and } 14 \\
\text { months. }\end{array}$ & $\begin{array}{l}\text { Design phase: } \\
\text { N/A } \\
\text { Test phase: } \\
\text { - Questionnaires } \\
\text { - Observation/ } \\
\text { experimenter log } \\
\text { journal } \\
\text { - Data log } \\
\text { Results: } \\
\text { Authors conclude: } \\
\text { Participants were able to } \\
\text { use the app } \\
\text { efficiently, and it } \\
\text { facilitated their } \\
\text { day-to-day activities. }\end{array}$ & $\begin{array}{l}\text { Individualized } \\
\text { programme } \\
\text { with training } \\
\text { session } \\
\text { based on } \\
\text { cognitive } \\
\text { rehabilitation } \\
\text { methods. }\end{array}$ & N/A \\
\hline
\end{tabular}




\section{Table 2 Continued}

\begin{tabular}{|c|c|c|c|c|c|c|c|c|}
\hline \multirow[b]{3}{*}{ STUDY } & & \multicolumn{2}{|c|}{ REVIEW AIM 1} & \multicolumn{3}{|c|}{ REVIEW AIM 2} & \multicolumn{2}{|c|}{ REVIEW AIM 3} \\
\hline & \multirow[b]{2}{*}{$\begin{array}{l}\text { PUBLICATION } \\
\text { AND ORIGIN }\end{array}$} & \multicolumn{2}{|c|}{$\begin{array}{c}\text { ASSISTIVE TECHNOLOGY USED BY } \\
\text { PEOPLE WITH DEMENTIA FOR } \\
\text { SELF-MANAGEMENT }\end{array}$} & \multicolumn{3}{|c|}{$\begin{array}{l}\text { INVOLVEMENT OF END USERS IN } \\
\text { DESIGN AND/OR TEST PHASES }\end{array}$} & \multicolumn{2}{|c|}{$\begin{array}{l}\text { METHODS ADDRESSING } \\
\text { ADOPTION AND } \\
\text { DISSEMINATION }\end{array}$} \\
\hline & & $\begin{array}{l}\text { TECHNOLOGY } \\
\text { AND TARGET } \\
\text { USERS }\end{array}$ & FEATURES & PARTICIPANTS & $\begin{array}{l}\text { METHODS } \\
\text { FOR USER } \\
\text { INVOLVEMENT }\end{array}$ & $\begin{array}{l}\text { EVALUATION } \\
\text { METHODS AND } \\
\text { RESULTS }\end{array}$ & ADOPTION & DISSEMINATION \\
\hline Alzminder & $\begin{array}{l}\text { Xenakidis et al. } \\
\quad(2014) \\
\text { Origin: Cyprus }\end{array}$ & $\begin{array}{l}\text { Aim: Support } \\
\text { various needs } \\
\text { across multiple } \\
\text { cognitive } \\
\text { domains. } \\
\text { Target users: } \\
\text { people with } \\
\text { cognitive decline, } \\
\text { including people } \\
\text { with dementia. }\end{array}$ & $\begin{array}{l}\text { The holistic } \\
\text { app was } \\
\text { designed for } \\
\text { smartphones } \\
\text { and included: } \\
\text { - Reminders } \\
\text { - Music } \\
\text { therapy and } \\
\text { entertainment } \\
\text { - Photos } \\
\text { with narratives } \\
\text { - Games } \\
\text { - Contact details } \\
\text { Automatic call } \\
\text { and SMS to } \\
\text { emergency } \\
\text { contacts } \\
\text { Test phase: } \\
\text { Prototype testing } \\
\text { was planned } \\
\text { (access to the } \\
\text { app on Google Play). }\end{array}$ & $\begin{array}{l}\text { Design phase: } \\
\text { Professionals, } \\
\text { stakeholders and } \\
\text { people with MCI } \\
\text { (numbers not } \\
\text { specified). } \\
\text { Specific details } \\
\text { verifying } \\
\text { diagnosis: No }\end{array}$ & $\begin{array}{l}\text { Design phase: } \\
\text { A prototype was } \\
\text { designed based } \\
\text { on the analysis } \\
\text { of already } \\
\text { existing } \\
\text { technologies } \\
\text { promoted for } \\
\text { people with } \\
\text { dementia. } \\
\text { The prototype was } \\
\text { demonstrated } \\
\text { during } \\
\text { consultations. }\end{array}$ & N/A & $\begin{array}{l}\text { Audio-based } \\
\text { guidance } \\
\text { was included } \\
\text { in the app. }\end{array}$ & N/A \\
\hline
\end{tabular}


Table 2 Continued

\begin{tabular}{|c|c|c|c|c|c|c|c|c|}
\hline \multirow[b]{3}{*}{ STUDY } & \multirow[b]{3}{*}{$\begin{array}{l}\text { PUBLICATION } \\
\text { AND ORIGIN }\end{array}$} & \multicolumn{2}{|c|}{ REVIEW AIM 1} & \multicolumn{3}{|c|}{ REVIEW AIM 2} & \multicolumn{2}{|c|}{ REVIEW AIM 3} \\
\hline & & \multicolumn{2}{|c|}{$\begin{array}{c}\text { ASSISTIVE TECHNOLOGY USED BY } \\
\text { PEOPLE WITH DEMENTIA FOR } \\
\text { SELF-MANAGEMENT }\end{array}$} & \multicolumn{3}{|c|}{$\begin{array}{l}\text { INVOLVEMENT OF END USERS IN } \\
\text { DESIGN AND/OR TEST PHASES }\end{array}$} & \multicolumn{2}{|c|}{$\begin{array}{c}\text { METHODS ADDRESSING } \\
\text { ADOPTION AND } \\
\text { DISSEMINATION }\end{array}$} \\
\hline & & $\begin{array}{l}\text { AIM OF } \\
\text { TECHNOLOGY } \\
\text { AND TARGET } \\
\text { USERS }\end{array}$ & FEATURES & PARTICIPANTS & $\begin{array}{l}\text { METHODS } \\
\text { FOR USER } \\
\text { INVOLVEMENT }\end{array}$ & $\begin{array}{l}\text { EVALUATION } \\
\text { METHODS AND } \\
\text { RESULTS }\end{array}$ & ADOPTION & DISSEMINATION \\
\hline $\begin{array}{l}\text { Digital } \\
\text { prompter }\end{array}$ & $\begin{array}{l}\text { Boyd et al. } \\
\quad(2017) \\
\text { Origin: } \\
\text { United } \\
\text { Kingdom }\end{array}$ & $\begin{array}{l}\text { Aim: Guide the } \\
\text { independent } \\
\text { performance } \\
\text { of multistep } \\
\text { tasks at home. } \\
\text { Target users: } \\
\text { people with } \\
\text { dementia. }\end{array}$ & $\begin{array}{l}\text { The app was } \\
\text { designed for a } \\
\text { tablet computer. } \\
\text { It provided } \\
\text { personalized } \\
\text { prompts (text, } \\
\text { audio messages } \\
\text { and photos) on } \\
\text { how to perform } \\
\text { daily tasks. } \\
\text { The prompts were } \\
\text { loaded into the } \\
\text { system by the family } \\
\text { caregiver through } \\
\text { separate software. }\end{array}$ & $\begin{array}{l}\text { Design phase: } \\
\text { Authors refer to } \\
\text { user engagement } \\
\text { (not specified). } \\
\text { Test phase: } \\
12 \text { dyads: PwD and } \\
\text { FC. } \\
\text { Specific details } \\
\text { verifying } \\
\text { diagnosis: No. }\end{array}$ & $\begin{array}{l}\text { Test phase: } \\
\text { Home } \\
\text { testing. } \\
\text { Duration: } \\
5 \text { weeks }\end{array}$ & $\begin{array}{l}\text { Test phase: } \\
\text { Semistructured } \\
\text { interviews } \\
\text { Results: Authors } \\
\text { conclude: Participants } \\
\text { and caregivers could } \\
\text { to some extend operate } \\
\text { the system in a home- } \\
\text { based setting, but with } \\
\text { varied success. }\end{array}$ & $\begin{array}{l}\text { Training } \\
\text { session and } \\
\text { a manual for } \\
\text { caregivers. } \\
\text { Telephone } \\
\text { support and } \\
\text { hotline. }\end{array}$ & N/A \\
\hline \multirow[t]{2}{*}{$\begin{array}{l}\text { Video } \\
\text { reminding } \\
\text { technology }\end{array}$} & $\begin{array}{l}\text { Donnelly } \\
\text { et al. }(2010) \\
\text { Origin: } \\
\text { Northern } \\
\text { Ireland }\end{array}$ & \multirow[t]{2}{*}{$\begin{array}{l}\text { Aim: Provide } \\
\text { individualized } \\
\text { video-based } \\
\text { reminders to } \\
\text { support memory. } \\
\text { Target users: people } \\
\text { with dementia. }\end{array}$} & \multirow[t]{2}{*}{$\begin{array}{l}\text { The personalized } \\
\text { video-based } \\
\text { reminders were } \\
\text { delivered on a } \\
\text { modified mobile } \\
\text { phone. } \\
\text { The video } \\
\text { reminders were } \\
\text { created by } \\
\text { caregivers and } \\
\text { loaded through } \\
\text { separate software. }\end{array}$} & $\begin{array}{l}\text { Design phase: } \\
\text { PwD and FC } \\
\text { Pretrial test for } \\
\text { adoption of } \\
\text { the AT: } \\
\text { Four dyads: PwD } \\
\text { and FC, } 5 \\
\text { controls. } \\
\text { Specific details } \\
\text { verifying } \\
\text { diagnosis: Yes }\end{array}$ & $\begin{array}{l}\text { Design phase: } \\
\text { Interviews } \\
\text { Pretrial test } \\
\text { phase: } \\
\text { Home testing } \\
\text { Duration: } 3 \\
\text { days }\end{array}$ & $\begin{array}{l}\text { Pretrial test phase: } \\
\text { Interviews. } \\
\text { Results: The prototype was } \\
\text { refined based on the } \\
\text { results from this test } \\
\text { phase. }\end{array}$ & N/A & N/A \\
\hline & $\begin{array}{l}\text { Nugent et al. } \\
\text { (2011) } \\
\text { Origin: } \\
\text { Northern } \\
\text { Ireland }\end{array}$ & & & $\begin{array}{l}\text { Test phase: } \\
\text { Four dyads: } \\
\text { PwD and FC. } \\
\text { Specific details } \\
\text { verifying } \\
\text { diagnosis: No. } \\
\text { but from a } \\
\text { memory clinic. }\end{array}$ & $\begin{array}{l}\text { Test phase: } \\
\text { Home testing } \\
\text { Duration: } \\
5 \text { weeks. }\end{array}$ & $\begin{array}{l}\text { Test phase: } \\
\text { - Questionnaires. } \\
\text { - Data log registering } \\
\text { the use of the } \\
\text { system } \\
\text { Results: Authors conclude: } \\
\text { The technology was } \\
\text { usable, and caregiver's } \\
\text { involvement was } \\
\text { essential. }\end{array}$ & $\begin{array}{l}\text { One } \\
\text { individualised } \\
\text { training } \\
\text { session for } \\
\text { participant } \\
\text { and caregiver } \\
\text { dyads. }\end{array}$ & N/A \\
\hline
\end{tabular}


Table 2 Continued

\begin{tabular}{|c|c|c|c|c|c|c|c|c|}
\hline \multirow[b]{3}{*}{ STUDY } & \multirow[b]{3}{*}{$\begin{array}{l}\text { PUBLICATION } \\
\text { AND ORIGIN }\end{array}$} & \multicolumn{2}{|c|}{ REVIEW AIM 1} & \multicolumn{3}{|c|}{ REVIEW AIM 2} & \multicolumn{2}{|c|}{ REVIEW AIM 3} \\
\hline & & \multicolumn{2}{|c|}{$\begin{array}{c}\text { ASSISTIVE TECHNOLOGY USED BY } \\
\text { PEOPLE WITH DEMENTIA FOR } \\
\text { SELF-MANAGEMENT }\end{array}$} & \multicolumn{3}{|c|}{$\begin{array}{l}\text { INVOLVEMENT OF END USERS IN } \\
\text { DESIGN AND/OR TEST PHASES }\end{array}$} & \multicolumn{2}{|c|}{$\begin{array}{c}\text { METHODS ADDRESSING } \\
\text { ADOPTION AND } \\
\text { DISSEMINATION }\end{array}$} \\
\hline & & $\begin{array}{l}\text { TECHNOLOGY } \\
\text { AND TARGET } \\
\text { USERS }\end{array}$ & FEATURES & PARTICIPANTS & $\begin{array}{l}\text { METHODS } \\
\text { FOR USER } \\
\text { INVOLVEMENT }\end{array}$ & $\begin{array}{l}\text { EVALUATION } \\
\text { METHODS AND } \\
\text { RESULTS }\end{array}$ & ADOPTION & DISSEMINATION \\
\hline KITE & $\begin{array}{l}\text { Robinson et al. } \\
\text { (2009) } \\
\text { Origin: United } \\
\text { Kingdom }\end{array}$ & $\begin{array}{l}\text { Aim: facilitate } \\
\text { independence. } \\
\text { Target users: } \\
\text { people with } \\
\text { dementia. }\end{array}$ & $\begin{array}{l}\text { Prototypes of two } \\
\text { individualized } \\
\text { technologies: } \\
\text { - Electronic } \\
\text { reminder } \\
\text { including GPS } \\
\text { tracking. } \\
\text { - Armband } \\
\text { with pedometer } \\
\text { and GPS } \\
\text { tracking. }\end{array}$ & $\begin{array}{l}\text { Design phase: } \\
10 \text { PwD, } 11 \\
\text { FC, } 4 \text { volunteers. } \\
\text { Specific details } \\
\text { verifying } \\
\text { diagnosis: No, } \\
\text { but from } \\
\text { dementia care } \\
\text { organizations. } \\
\text { Test phase: } \\
\text { N/A }\end{array}$ & $\begin{array}{l}\text { Design phase: } \\
\text { Workshops } \\
\text { and } \\
\text { consultations. }\end{array}$ & $\begin{array}{l}\text { Design phase: } \\
\text { End users } \\
\text { feedback on } \\
\text { prototypes. }\end{array}$ & N/A & N/A \\
\hline $\begin{array}{l}\text { Autonomous } \\
\text { spatial } \\
\text { navigation }\end{array}$ & $\begin{array}{l}\text { Lanza et al. } \\
\quad(2014) \\
\text { Origin: } \\
\text { Germany }\end{array}$ & $\begin{array}{l}\text { Aim: support } \\
\text { autonomous } \\
\text { spatial navigation } \\
\text { Target users: people } \\
\text { with dementia. }\end{array}$ & $\begin{array}{l}\text { The navigation } \\
\text { system integrated } \\
\text { photo-based } \\
\text { navigation with } \\
\text { acoustic and } \\
\text { optic feedback. } \\
\text { It was designed } \\
\text { to be used on a } \\
\text { smartphone. }\end{array}$ & $\begin{array}{l}\text { Design phase: } \\
\text { No involvement } \\
\text { of people with } \\
\text { dementia. } \\
\text { Test phase: } \\
14 \text { participants } \\
\text { with Alzheimer's } \\
\text { disease. } \\
\text { Specific details } \\
\text { verifying diagnosis } \\
\text { of participants: } \\
\text { Yes }\end{array}$ & $\begin{array}{l}\text { Design phase: } \\
\text { N/A. } \\
\text { Test phase: } \\
\text { Outdoor } \\
\text { laboratory } \\
\text { pilot test at a } \\
\text { hospital } \\
\text { campus. } \\
\text { Duration: } \\
\text { One test } \\
\text { session }\end{array}$ & $\begin{array}{l}\text { Registration of participants' } \\
\text { behavior, e.g. time to } \\
\text { complete navigation task } \\
\text { and number of errors. } \\
\text { Results: } \\
\text { Authors conclude: } \\
\text { Participants perform } \\
\text { better when using the } \\
\text { navigation technology. }\end{array}$ & $\begin{array}{l}\text { Short } \\
\text { individual } \\
\text { instruction. }\end{array}$ & N/A \\
\hline
\end{tabular}

N/A: Not available, the study does not provide information on this topic.

Abbreviations: $\mathrm{AD}$, Alzheimer's disease; MCI, mild cognitive impairment; PwD, people with dementia (not specified); FC, family caregiver. 


\section{Results}

Eleven papers derived from eight studies fulfilled the initial inclusion criteria and were included in the review. Three of the studies presented complementary data from the design and the test phase in two separate papers. The results are summarized in Table 2, including specific characteristics related to the three aims of this scoping review.

\section{Review aim 1: AT designed to support self- management of people with dementia}

All studies included in this review addressed technology that was designed to support people with dementia in ways related to self-management. The aims and features of these technologies varied widely. Two studies, COGKNOW (Meiland et al., 2007; Meiland et al., 2012), and Rosetta (Hattink et al., 2016; Meiland et al., 2014), were comprehensive multidevice holistic solutions, addressing a variety of functional domains including memory, social contacts, daily activities, and safety. These studies were also related, as the COGKNOW solution was integrated in the Rosetta solution. Two studies, AP@LZ (Imbeault et al., 2014), and Alzminder (Xenakidis et al., 2014), presented holistic app solutions comprising a variety of features that could support the user in everyday life, e.g. reminders, information on contacts, medication information, or emergency contacts. In two other studies, Digital Prompter (Boyd et al., 2017) and Video Reminding Technology (Donnelly et al., 2010; Nugent et al., 2011), the technology was designed with one main feature that was intended to support the user in various home-bound situations. The last two studies, KITE (Robinson et al., 2009) and Autonomous Spatial Navigation (Lanza et al., 2014), described technology solutions addressing one specific functional domain, in these cases support of independent outdoor navigation.

\section{Review aim 2: Involving people with dementia in the design and/or test of the AT}

There was a great variety among studies when it came to involvement of people with dementia in the design phase. Three of the studies (Meiland et al., 2007; Meiland et al., 2014; Robinson et al., 2009) based the design of technology on an extensive iterative design process involving people with dementia, family caregivers, dementia professionals, volunteers, and other stakeholders. Methods used in these design phases were mainly workshops, interviews, and consultations. The number of people with dementia involved in these design phases varied between 10 and 27. Two studies (Boyd et al., 2017; Donnelly et al., 2010) referred to involvement of end users and family caregivers in the design phase, but they did not provide any specific details on the number of participants or specific methods of involvement. However, Donnelly et al. involved a small group of end users in a prototype pretest. Xenakidis et al. (2014) designed their prototype app based on the analysis of already existing technology, and they consulted stakeholders and people with mild cognitive disorder. They did not report any specific details of this involvement or indicate if it had any impact on the design of their app. Two studies (Imbeault et al., 2014; Lanza et al., 2014) did not involve any end users or other stakeholders in the design of their technology solutions.

Six of the studies involved people with dementia in a test phase. The methods applied, number of participants involved, and the duration of the test period varied greatly. Lanza et al. (2014) conducted a one-session outdoor laboratory test where they assessed the performance of 14 participants when they used technology to support navigation compared with when they used a map. Imbeault et al. (2014) included two participants in the testing phase; however, they were involved in a quite extensive program of laboratory and home testing for 11 and 14 months, respectively. Four other studies also involved home testing. Boyd et al. (2017) included 4 dyads of people with dementia and a family caregiver, and Nugent et al. (2011) included 12 dyads. Both studies had a test period of 5 weeks. Meiland et al. (2012) included 42 dyads of people with dementia and a family caregiver in three test cycles that lasted between 0.5 days and 8 weeks. Hattink et al. (2016) included 42 participants in a controlled trial where the Rosetta system was tested at home for a variable period ranging from 0.5 to 8 months.

Methods for data collection from these test phases varied among these six studies. Lanza et al. (2014) collected observational data describing participants' behavior during the laboratory test. The remaining five studies (Boyd et al., 2017; Hattink et al., 2016; Imbeault et al., 2014; Meiland et al., 2012; Nugent et al., 2011) all collected qualitative data mainly from interviews and questionnaires, but other methods were also used, e.g. observations or the use of a diary (Hattink et al., 2016; Imbeault et al., 2014). In addition, Imbeault et al. (2014), Meiland et al. (2012), and Nugent et al. (2011) used data logs to register the use of technology. These data were generally used to assess usability and user-friendliness of the technology. Meiland et al. (2012) and Hattink et al. (2016) also used standardized outcome measures, addressing, e.g. quality of life, coping and autonomy, to measure effectiveness of the technology. Meiland et al. (2012) compared the pre- and posttest performance of a subgroup of participants, and Hattink et al. (2016) 
compared between group differences on pre- and posttest performance in their controlled trial. Neither of the studies found statistically significant differences on any outcome measures.

Due to the heterogeneous nature of the outcome measures used and results presented in the studies, and according to the review aims of exploring methodological issues, the specific results of the studies are not summarized here.

Two studies (Robinson et al., 2009; Xenakidis et al., 2014) did not include a specific test phase in their publications, but Robinson et al. (2009) included feedback from two end users and one family caregiver during the design of their prototypes. The Alzminder app (Xenakidis et al., 2014) has been made available as open access on Google Play (2019) for prototype testing. We did not locate any scientific or other publications related to this test phase.

\section{Review aim 3: Dissemination and adoption of AT for people with dementia}

Five of the studies provided information on methods used to support people with dementia in adopting the technology during the test phase. The most extensive method was applied by Imbeault et al. (2014), who conducted an individualized program with training sessions based on the established cognitive rehabilitation methods. Meiland et al. (2012) provided a user manual and helpdesk support. Two studies (Hattink et al., 2016; Nugent et al., 2011) conducted individual training sessions with participants and caregivers, and Lanza et al. (2014) gave participants a short instruction before conducting their outdoor laboratory tests. One study (Boyd et al., 2017) only addressed caregivers with training sessions and telephone support. In addition, Xenakidis et al. (2014) included audio-based guidance as part of their design of the app.

All included studies described technology solutions that were developed to a prototype level, and it was not possible to locate additional information that indicated whether these solutions had been further developed or disseminated. Even though the COGKNOW solution (Meiland et al., 2012) was further developed to be included in the Rosetta project (Meiland et al., 2014), this was still at a prototype level.

\section{Discussion}

This scoping review was conducted to investigate the current state of research addressing AT designed to be used by people with dementia for selfmanagement. It explored how user involvement was addressed in the design and test of such usercentered technology and how dissemination and adoption of the solutions was approached.

The eight studies that fulfilled the inclusion criteria represented a broad and varied field of AT solutions, ranging from comprehensive multidevice holistic solutions, holistic app solutions, technology designed to support daily living through one main feature, and solutions addressing one specific functional domain. The limited number of studies identified reveals that a relatively little amount of the research activity within the field of AT and dementia is directed at creating solutions that can be used by people with dementia for self-management. It could also indicate that AT solutions promoted as applicable for self-management of people with dementia are not based on research.

\section{User involvement}

User involvement in the design and test of technologies varied greatly among the studies included in this review, ranging from no involvement of end users or other stakeholders to extensive user involvement throughout an iterative design process. The methods applied for user involvement in testing and the extent of testing also differed greatly, ranging from a one-session outdoor laboratory testing to extensive single case studies. Most studies involved testing in the homes of end users.

The heterogeneous approach to user involvement, and in some cases lack of user involvement, has also been discussed in other reviews (Ienca et al., 2017; Meiland et al., 2017). Our results underline the need to validate methods and create guidelines for the involvement of people with dementia in designing, testing, and conducting research on AT. Established models for user-centered design (Gulliksen et al., 2003) should be adapted to be used with people with dementia. Previous examples of successful user involvement should of course also be drawn on, as represented in this review by Meiland et al. (2007), Meiland et al. (2014), and Robinson et al. (2009). Alzheimer Europe's position paper on involving people with dementia in research through patient and public involvement (Gove et al., 2018) highlights potential challenges and benefits associated with such involvement and could be a benchmark for developing such guidelines.

\section{Adoption and dissemination of AT}

Adoption was generally addressed in the studies, but in very different ways, and the methods applied were only targeting the context of prototype testing. None of the studies reflected on the applicability and effectiveness of these adoption methods or how they could be used for future adoption of 
technology. Dissemination of technology was not discussed in any of the studies.

These results are in line with results from other studies demonstrating that dissemination and adoption of AT by people with dementia have only to a limited extent been addressed in research (Gibson et al., 2018; Knapp et al., 2015), and it underlines the need to develop evidence-based methods for adoption and dissemination. Moreover, the results also demonstrate that this research field is characterized by a quite narrow focus on the design process and lacks a holistic approach to the dynamic interrelationship between technology, user, and context. Van Gemert-Pijnen et al. (2011) developed an evidence-based holistic framework that can serve as a guideline for development, implementation, and evaluation of e-Health technologies. This framework incorporates user-centered design, participatory development, and test, as well as dissemination, adoption, and evaluation of the actual uptake of technologies. It also addresses the need to include business case models to reinforce viable technology solutions. The framework also underlines the obvious need to involve a variety of stakeholders to ensure that all these aspects are adequately included and operationalized when developing technologies. This holistic and agile framework seems highly suitable to be applied when developing AT for people with dementia (van Gemert-Pijnen and Span, 2017). It could reinforce the involvement of a variety of stakeholders and experts to ensure that all relevant aspects are met throughout the entire life cycle of AT for people with dementia, from scoping and initial design to dissemination and adoption.

\section{Study design, methods, and evidence}

As described, the results of this review were generally characterized by a considerable variability among studies on all parameters. These profound variations draw a picture of a heterogeneous and fragmented field of research, dominated by smallscale studies that mainly address the design and test of AT prototypes. None of the studies were designed or powered to provide evidence for the effectiveness of technology. Hence, our results underline that there is a general need for suitable and applicable research methodology that can provide higher levels of evidence.

Within the related field of digital health technologies, encompassing apps, programs, and software used in the health and care system (The National Institute for Health and Care Excellence (2018), there is an ongoing debate on how to address research methodology and evidence (Greaves et al., 2018). The dynamic and iterative nature of such technologies and the cost of research compared with a product's perceived level of risk are often used to argue against the use of traditional comparative methods to provide evidence and to reject randomized controlled trials as the gold standard. Opposite to this, there is a growing demand that such technologies should not be excepted from provid ing evidence to document clinical- and costeffectiveness, and standards relevant for this type of intervention, e.g. standards for data security, data management, and ethics (Greaves et al., 2018).

Several initiatives have addressed the need for standardized procedures when conducting and reporting research related to digital health technologies. Recently, the National Institute for Health and Care Excellence published a framework for evidence of effectiveness standards and economic impact standards for digital health technologies (The National Institute for Health and Care Excellence, 2018). We find that AT supporting self-management of people with dementia can be stratified within this framework, and the minimum evidence standards outlined in this framework can serve as guidelines when designing research within this field. The AT industry, which promotes solutions as applicable for people with dementia, should of course also comply with the standards. Incorporating such evidence standards would be a great benefit for this field of research and could promote a more transparent market of evidence-based AT solutions for people with dementia.

We also observed a lack of standards for conducting and reporting trials among the studies included in this review. An extension of the Consolidated Standards of Reporting Trials addressing e-health solutions, CONSORT-EHEALTH, was published in 2011 (Eysenbach and ConsortEHEALTH, 2011), and we suggest that this standard can be applied in this field of research since the concept of e-Health as defined by WHO (2005) as "the use of information and communication technologies (ICT) for health" is in many instances coterminous with electronic AT. The implementation of standards on conducting and reporting research could also bring this field of research a great step forward.

Moreover, during the review of full-text records, 10 papers were excluded because only preliminary ideas or drafting of AT solutions were reported, no data documented the development or test of an operational prototype. Despite our effort to search additional information, including grey literature, no supplementary data were identified. There can of course be various reasons for this, e.g. lack of funding. Another possible reason is suppression or a tendency not to publish "negative results" describing details of studies that did not result in successful 
design of an AT solution. Such possible publication bias is a severe limitation when comparing and synthesizing data from various studies and aiming for a balanced perspective on this field of research. There is also a risk that it can influence funders, policymakers, and skew industry influence if all potential benefits and limitations of designing AT for people with dementia are not thoroughly disclosed.

\section{Outcome measures}

The results of this review also indicate that the low level of evidence is influenced by the lack of standardized outcome measures to assess usability, effectiveness, and benefits of AT for people with dementia.

The studies included in this review mainly relied on qualitative results from workshops, interviews, and questionnaires specifically developed for the study. Such methods are of course essential when collecting qualitative data, and it can provide crucial data during a user-involving iterative design process as demonstrated by three of the studies in this review (Meiland et al., 2007; Meiland et al., 2014 Robinson et al., 2009). However, the use of standardized outcome measures when testing technology and assessing usability and effectiveness would increase the reliability of results and enable comparison of studies and research results. The development and validation of such outcome measures could benefit from research in psychosocial interventions for people with dementia, where efforts are made to create outcome measures that capture the essence of such interventions (Øksnebjerg et al., 2018). The development of AT outcome measures could find inspiration in the theoretical and methodology advances within the field of psychosocial research, where there is a growing focus on positive psychology outcomes (Stoner et al., 2015; Wolverson et al., 2016), to introduce outcome measures that reflect interactions between AT use, positive psychology. and self-management of people with dementia (Serino and Pedroli, 2016).

Another dimension of outcome measures in AT research is the potential of using log data to assess applicability, usability, and adoption of technology. Three studies (Imbeault et al., 2014; Meiland et al., 2012; Nugent et al., 2011) used log data among their outcome measures, but none of the studies gave any specific attention to these results and the potential of using such outcome measures. This is in contrast to the increasing use of log data and big data within healthcare and disease management (Ienca et al., 2018; Sieverink et al., 2017), and the potential of using $\log$ data to assess usability, effectiveness and adherence to AT should be addressed in future studies.

\section{Conclusion and perspectives}

The results of this scoping review underline the lack of well-designed high-quality research that can establish evidence-based methods on how to deliver user-centered AT solutions for people with dementia and provide evidence that AT can be effective to support self-management.

The results demonstrate that this field of research is in need of guidelines and evidence-based methods to promote and qualify user involvement. The results also emphasize the need for research into all aspects that are essential to deliver applicable, effective, and sustainable AT solutions to people with dementia. Future research has to go beyond the stages of design and testing to provide more knowledge and evidence-based methods for dissemination and adoption.

Moreover, it could be highly beneficial to implement standards for conducting and reporting research, to improve study design, level of evidence, and impact of research results. It would also be highly beneficial for the quality of research and level of evidence if standardized outcome measures that capture the interaction of AT and self-management of people with dementia were developed and applied.

These perspectives should be further elaborated and incorporated in future studies addressing AT for people with dementia.

\section{Conflict of interest}

None.

\section{Description of authors' roles}

The first author was involved in all aspects of conceptualizing and design of the scoping review and the collection, analysis, and interpretation of data. The second author was involved in data collection and analysis. All authors were involved in writing the manuscript, and all authors have read and approved the final manuscript.

\section{Acknowledgements}

Danish Dementia Research Centre is supported by the Danish Ministry of Health. 


\section{References}

Alzheimer's Disease International. (2016). Bibliography of References to National Plans. Available at: https://www.alz. co.uk/sites/default/files/pdfs/national-plans-bibliography2016.pdf; accessed 1 February 2019.

Asghar, I., Cang, S. and Yu, H. (2017). Assistive technology for people with dementia: an overview and bibliometric study. Health Information \& Libraries fournal, 34, 5-19. doi: 10.1111/hir.12173.

Assistive Technology Industry Association. (2019). What is AT? - Assistive Technology Industry Association. Available at: http://www.atia.org/at-resources/what-is-at/; accessed 1 February 2019.

Bächle, M., Daurer, S., Judt, A. and Mettler, T. (2018). Assistive technology for independent living with dementia: stylized facts and research gaps. Health policy and technology, 7, 98-111. doi: 10.1016/j.hlpt.2017.12.002.

Barlow, J., Wright, C., Sheasby, J., Turner, A. and Hainsworth, J. (2002). Self-management approaches for people with chronic conditions: a review. Patient Education and Counseling, 48, 177-187. doi: 10.1016/S0738-3991(02) 00032-0.

Boyd, H., Evans, N., Cheston, R., Noonan, K. and Harris, N. (2017). Home testing of a digital prompter for people with dementia. Studies in Health Technology and Informatics, 242, 27-30. doi: 10.3233/978-1-61499-798-6-27.

Covidence.org. Available at: https://www.covidence.org/ home, accessed 2018.

Donnelly, M. P. et al. (2010). A mobile multimedia technology to aid those with Alzheimer's disease. IEEE multimedia, 17, 42-51. doi: 10.1109/MMUL.2010.25.

Eysenbach, G. and Consort-EHEALTH (2011). CONSORT-EHEALTH: improving and standardizing evaluation reports of Web-based and mobile health interventions. Fournal of Medical Internet Research, 13, e126. doi: 10.2196/jmir.1923.

Federici, S., Meloni, F. and Borsci, S. (2016). The abandonment of assistive technology in Italy: a survey of users of the national health service. European fournal of Physical and Rehabilitation Medicine, 52, 516-526.

Gibson, G., Dickinson, C., Brittain, K. and Robinson, L. (2015). The everyday use of assistive technology by people with dementia and their family carers: a qualitative study. BMC Geriatrics, 15, 89. doi: 10.1186/s8877-015-0091-3.

Gibson, G., Dickinson, C., Brittain, K. and Robinson, L.A. (2019). Personalisation, customisation and bricolage: how people with dementia and their families make assistive technology work for them. Ageing $\mathcal{E}$ Society, 39, 2502-2519. doi: $10.1017 /$ S0144686X18000661.

Gibson, G., Newton, L., Pritchard, G., Finch, T., Brittain, K. and Robinson, L. (2016). The provision of assistive technology products and services for people with dementia in the United Kingdom. Dementia, 15, 681-701. doi: $10.1177 / 1471301214532643$.

Gillespie, A., Best, C. and O'Neill, B. (2012). Cognitive function and assistive technology for cognition: a systematic review. Fournal of the International Neuropsychological Society, 18, 1-19. doi: 10.1017/S1355617711001548.

GooglePlay. 2019. Available at: https://play.google.com/ store/apps/details?id=com.alzminder.mobilelite\&hl=en; accessed 1 February 2019.
Gove, D. et al. (2018). Alzheimer Europe's position on involving people with dementia in research through PPI (patient and public involvement). Aging \& Mental Health, 22, 723-729. doi: 10.1080/13607863.2017.1317334.

Greaves, F., Joshi, I., Campbell, M., Roberts, S., Patel, N. and Powell, J. (2018). What is an appropriate level of evidence for a digital health intervention? The Lancet. doi: 10.1016/S0140-6736(18)33129-5.

Gulliksen, J., Göransson, B., Boivie, I., Blomkvist, S., Persson, J. and Cajander, Å. (2003). Key principles for user-centred systems design. Behaviour and Information Technology, 22, 397-409. doi: 10.1080/ 01449290310001624329.

Hattink, B. et al. (2016). The electronic, personalizable Rosetta system for dementia care: exploring the userfriendliness, usefulness and impact. Disability and Rehabilitation: Assistive Technology, 11, 61-71. doi: 10.3109/ 17483107.2014.932022.

Holthe, T., Halvorsrud, L., Karterud, D., Hoel, K.-A. and Lund, A. (2018). Usability and acceptability of technology for community-dwelling older adults with mild cognitive impairment and dementia: a systematic literature review. Clinical Interventions in Aging, 13, 863. doi: 10 .2147/CIA.S154717.

Ienca, M. et al. (2017). Intelligent assistive technology for Alzheimer's disease and other dementias: a systematic review. Fournal of Alzheimer's Disease, 56, 1301-1340. doi: 10.3233/JAD-179005.

Ienca, M., Ferretti, A., Hurst, S., Puhan, M., Lovis, C. and Vayena, E. (2018). Considerations for ethics review of big data health research: a scoping review. PLoS ONE, 13, e0204937. doi: 10.1371/journal.pone.0204937.

Imbeault, H. et al. (2014). Electronic organiser and Alzheimer's disease: fact or fiction? Neuropsychological Rehabilitation, 24, 71-100. doi: 10.1080/09602011.2013 .858641 .

Kenigsberg, P.-A. et al. (2017). Assistive technologies to address capabilities of people with dementia: from research to practice. Dementia, doi: 10.1177/1471301217714093.

King, A. C. and Dwan, C. (2017). Electronic memory aids for people with dementia experiencing prospective memory loss: a review of empirical studies. Dementia, doi: $10.1177 /$ 1471301217735180.

Knapp, M. et al. (2015). The case for investment in technology to manage the global costs of dementia. Available at: http://eprints.1se.ac.uk/66482/1/_lse.ac.uk_storage_ LIBRARY_Secondary_libfile_shared_repository_ Content_LSEE_The $\% 20$ case $\% 20$ for $\% 20$ investment $\%$ 20in\%20technology.pdf; accessed 1 February 2019.

Krebs, P. and Duncan, D.T. (2015). Health app use among US mobile phone owners: a national survey. FMIR mHealth and uHealth, 3, e101. doi: 10.2196/mhealth.4924.

Lanza, C., Knörzer, O., Weber, M. and Riepe, M.W. (2014). Autonomous spatial orientation in patients with mild to moderate Alzheimer's disease by using mobile assistive devices: a pilot study. Fournal of Alzheimer's Disease, 42, 879-884. doi: 10.3233/JAD-140063.

Meiland, F. et al. (2014). Participation of end users in the design of assistive technology for people with mild to severe cognitive problems; the European Rosetta project. International Psychogeriatrics, 26, 769-779. doi: 10.1017/ S1041610214000088. 
Meiland, F. et al. (2017). Technologies to support community-dwelling persons with dementia: a position paper on issues regarding development, usability, effectiveness and cost-effectiveness, deployment, and ethics. FMIR Rehabilitation and Assistive Technologies, 4. doi: 10 .2196/rehab.6376.

Meiland, F.J. et al. (2012). Usability of a new electronic assistive device for community-dwelling persons with mild dementia. Aging \& Mental Health, 16, 584-591. doi: 10 .1080/13607863.2011.651433.

Meiland, F.J. et al. (2007). COGKNOW: development of an ICT device to support people with mild dementia. fournal on Information Technology in Healthcare, 5, 166-177.

Nugent, C. et al. (2011). Evaluation of video reminding technology for persons with dementia. In International Conference on Smart Homes and Health Telematics (pp. 153-160). Berlin: Springer.

Øksnebjerg, L. et al. (2018). Towards capturing meaningful outcomes for people with dementia in psychosocial intervention research: a pan-European consultation. Health Expectations, 21, 1056-1065. doi: 10.1111/ hex.12799.

Peters, M.D., Godfrey, C.M., Khalil, H., McInerney, P., Parker, D. and Soares, C.B. (2015). Guidance for conducting systematic scoping reviews. International journal of evidence-based healthcare, 13, 141-146. doi: 10 .1097/XEB.0000000000000050.

Robillard, J.M., Cleland, I., Hoey, J. and Nugent, C. (2018). Ethical adoption: a new imperative in the development of technology for dementia. Alzheimer's $\mathcal{E}$ Dementia. doi: 10.1016/j.jalz.2018.04.012.

Robinson, L., Brittain, K., Lindsay, S., Jackson, D. and Olivier, P. (2009). Keeping In Touch Everyday (KITE) project: developing assistive technologies with people with dementia and their carers to promote independence. International Psychogeriatrics, 21, 494-502. doi: 10.1017/ S1041610209008448.

Serino, S. and Pedroli, E. (2016). Technology and cognitive empowerment for healthy elderly: the link between cognitive skills acquisition and well-being. In: D. Villani, P. Cipresso, A. Gaggioli and G. Riva (Eds.), Integrating Technology in Positive Psychology Practice (pp. 193-213). Hershey, PA: IGI Global.

Sieverink, F., Kelders, S., Poel, M. and van Gemert-Pijnen, L. (2017). Opening the black box of electronic health: collecting, analyzing, and interpreting log data. FMIR Research Protocols, 6. doi: 10.2196/ resprot.6452.

Smith, R.O. et al. (2018). Assistive technology products: a position paper from the first global research, innovation, and education on assistive technology (GREAT) summit. Disability and Rehabilitation: Assistive Technology, 1-13. doi: 10.1080/17483107.2018.1473895.
Stoner, C.R., Orrell, M. and Spector, A. (2015). Review of positive psychology outcome measures for chronic illness, traumatic brain injury and older adults: adaptability in dementia? Dementia and Geriatric Cognitive Disorders, 40, 340-357. doi: 10.1159/000439044.

The National Institute for Health and Care Excellence (NICE). 2018. Evidence Standards Framework for Digital Health Technologies. Available at: https://www.nice .org.uk/Media/Default/About/what-we-do/ourprogrammes/evidence-standards-framework/ digital-evidence-standards-framework.pdf; accessed 1 February 2019.

Tricco, A.C. et al. (2018). PRISMA extension for scoping reviews (PRISMA-ScR): checklist and explanation. Annals of Internal Medicine, 169, 467-473. doi: 10.7326/ M18-0850.

van der Roest, H.G., Wenborn, J., Pastink, C., Droes, R.M. and Orrell, M. (2017). Assistive technology for memory support in dementia. Cochrane Database of Systematic Reviews, 6, Cd009627. doi: 10.1002/14651858. CD009627.pub2.

van Gemert-Pijnen, J.E., Kelders, S.M. and Bohlmeijer, E.T. (2014). Understanding the usage of content in a mental health intervention for depression: an analysis of log data. Fournal of Medical Internet Research, 16. doi: 10.2196/jmir.2991.

van Gemert-Pijnen, J.E. et al. (2011). A holistic framework to improve the uptake and impact of eHealth technologies. fournal of Medical Internet Research, 13. doi: 10.2196/ jmir. 1672 .

van Gemert-Pijnen, L. and Span, M. (2017). CeHRes roadmap to improve dementia care. In: J. van Hoof, G. Demiris and E.J.M. Wouter (Eds.), Handbook of Smart Homes, Health Care and Well-Being (pp. 133-146). Switzerland: Springer.

Wolverson, E.L., Clarke, C. and Moniz-Cook, E.D. (2016). Living positively with dementia: a systematic review and synthesis of the qualitative literature. Aging $\mathcal{F}$ Mental Health, 20, 676-699. doi: 10.1080/13607863.2015. 1052777.

World Health Organisation (WHO) (2005). What is eHealth. Available at: https://www.who.int/goe/en/; accessed 25 June 2019.

World Health Organisation (WHO). (2017). Global action plan on the public health response to dementia 2017-2025. Available at: http://apps.who.int/iris/ bitstream/handle/10665/259615/?sequence $=1$; accessed 1 February 2019.

Xenakidis, C.N., Hadjiantonis, A.M. and Milis, G.M. (2014). A mobile assistive application for people with cognitive decline. In: 2014 International Conference on Interactive Technologies and Games (iTAG) (pp. 28-35). IEEE Computer Society. 